\title{
Ultrafast Band Structure Control of a Two-Dimensional Heterostructure
}

Søren Ulstrup, ${ }^{\dagger}$ Antonija Grubišić Čabo, ${ }^{\ddagger}$ Jill A. Miwa, ${ }^{\ddagger}$ Jonathon M. Riley, $₫$ Signe S. Grønborg, $\ddagger$ Jens C. Johannsen, ${ }^{\S}$ Cephise Cacho, "Oliver Alexander,,$\|$ Richard T. Chapman, " Emma Springate, $\|$ Marco Bianchi, ${ }^{\ddagger}$ Maciej Dendzik, Jeppe V. Lauritsen,, Phil D. C. King, " and Philip Hofmann*,‡

$\dagger$ Advanced Light Source, E. O. Lawrence Berkeley National Laboratory, Berkeley, California 94720, USA

$\ddagger$ Department of Physics and Astronomy, Interdisciplinary Nanoscience Center, Aarhus University, 8000 Aarhus C, Denmark

ףSUPA, School of Physics and Astronomy, University of St. Andrews, St. Andrews, United Kingdom

§Institute of Condensed Matter Physics, École Polytechnique Fédérale de Lausanne (EPFL), Switzerland

\|Central Laser Facility, STFC Rutherford Appleton Laboratory, Harwell, United Kingdom E-mail: philip@phys.au.dk 


\begin{abstract}
The electronic structure of two-dimensional (2D) semiconductors can be significantly altered by screening effects, either from free charge carriers in the material itself, or by environmental screening from the surrounding medium. The physical properties of $2 \mathrm{D}$ semiconductors placed in a heterostructure with other $2 \mathrm{D}$ materials are therefore governed by a complex interplay of both intra- and inter-layer interactions. Here, using time- and angle-resolved photoemission, we are able to isolate both the layer-resolved band structure and, more importantly, the transient band structure evolution of a model 2D heterostructure formed of a single layer of $\mathrm{MoS}_{2}$ on graphene. Our results reveal a pronounced renormalization of the quasiparticle gap of the $\mathrm{MoS}_{2}$ layer. Following optical excitation, the band gap is reduced by up to $\sim 400 \mathrm{meV}$ on femtosecond timescales due to a persistence of strong electronic interactions despite the environmental screening by the $n$-doped graphene. This points to a large degree of tuneability of both the electronic structure and electron dynamics for $2 \mathrm{D}$ semiconductors embedded in a van der Waals-bonded heterostructure.
\end{abstract}

KEYWORDS: Ultrafast time- and angle-resolved photoemission, band gap renormalization, 2D material heterostructures, graphene, transition metal dichalcogenides, $\mathrm{MoS}_{2}$. 
Van der Waals-bonded heterostructures of two-dimensional (2D) atomic sheets hold great promise for the bottom-up design of materials with new properties. ${ }^{1-3}$ Realising heterostructures with desired functionality, however, remains a formidable challenge. Environmental screening from neighbouring layers can severely modify the band structures of the individual 2D materials, even though no real chemical bonds are formed between them. ${ }^{4}$ In $2 \mathrm{D}$ semiconductors the quasiparticle band gap and the exciton binding energy have been observed to be strongly influenced both by the choice of substrate material and by excited electrons within the 2D material. ${ }^{5-8}$ When placing a single layer transition metal dichalcogenide (SL-TMDC) on a metallic substrate such as $\mathrm{Au}(111)$, a strong band gap renormalization is observed but the reduced band gap is almost unaffected by an additional excited carrier density. ${ }^{9,10}$ When the SL-TMDC is placed on a weakly screening insulator such as $\mathrm{SiO}_{2}$, the static band gap is close to that expected for the free-standing case ${ }^{11-13}$ but optically excited carriers can give rise to a giant band gap reduction. ${ }^{6}$ Such carrier-induced renormalization effects are somewhat similar to what is observed in bulk semiconductors ${ }^{14}$ and quantum wells, ${ }^{15,16}$ where they can be used to generate materials with non-linear optical properties. A key advantage of using a truly 2D semiconductor, however, is that its environment can be freely chosen, which should greatly enhance the tuneability of the system. We demonstrate this capability for a heterostructure of SL $\mathrm{MoS}_{2}$ and graphene $\left(\mathrm{MoS}_{2} / \mathrm{G}\right)$. Screening of the semiconductor by the adjacent graphene is expected to induce a moderate reduction of the quasiparticle band gap, ${ }^{4,17}$ yet we find that the $\mathrm{MoS}_{2}$ layer retains a strong optical tuneability. Using time- and angle-resolved photoemission (TR-ARPES), we not only directly visualise how this causes the band structure to change on femtosecond time scales following optical excitation of free charge carriers, but are also able to separate the underlying electronic structure and carrier dynamics from the SL $\mathrm{MoS}_{2}$ and the graphene.

In TR-ARPES, electrons are excited into unoccupied states using low-energy photons and then photoemitted with a time-delayed higher energy laser pulse. This type of experimental set-up provides information on the band structure and carrier population in the 
equilibrium and excited states, and on the time dependent population change after the initial excitation. For the material system and moderate excitation studied here, one does not typically expect the pump pulse to create changes in the band structure as such, but merely in the population of the states. This is indeed what we find for the graphene layer in our $\mathrm{MoS}_{2} / \mathrm{G}$ heterostructure: Electrons are pumped into the conduction band (CB) and follow a decay dynamics that closely resembles earlier findings for epitaxial graphene. ${ }^{18}$ On the other hand, the adjacent $\mathrm{MoS}_{2}$ bands are rigidly shifted in the presence of the excited carriers. We determine how these band shifts lead to a significant reduction of the quasiparticle band gap as a function of the excited carrier density, consistent with a recent theoretical calculation. ${ }^{5}$

\section{Results and Discussion}

The $\mathrm{MoS}_{2} / \mathrm{G}$ heterostructure was grown on a silicon carbide substrate by van der Waals epitaxy (see Materials and Methods section). Due to the weak interaction between the individual 2D layers the $\mathrm{MoS}_{2}$ grows with many rotational domains. However, our synthesis method yields a preference for $\mathrm{MoS}_{2}$ domains that can be rotated by $30^{\circ}$ and $90^{\circ}$ with respect to the underlying graphene (see sketch of the $30^{\circ}$ case in Figure 1a). As described in detail in Ref. 19 we can determine this rotational preference directly from ARPES scans of the overlapping BZs of the two materials since both the Dirac cone of graphene and the valence

band maximum (VBM) of SL $\mathrm{MoS}_{2}$ are found at the $\bar{K}$ point of their respective BZs (see Figure 1b). Note that since the electronic bands of $\mathrm{MoS}_{2}$ domains rotated by $30^{\circ}$ and $90^{\circ}$ overlap we can not distinguish such domains in ARPES. The rotation of the graphene and $\mathrm{MoS}_{2}$ BZs allows us to separately access the excited carrier dynamics around the Dirac cone in graphene and around the direct band gap of SL $\mathrm{MoS}_{2}$. Figure 1c provides the $\mathrm{MoS}_{2}$ valence band (VB) dispersion measured by ARPES with theoretical band structure calculations for the free-standing case overlaid. The Dirac cone of the underlying graphene is seen in Figure 1d. The Dirac point is found at $E_{D}=0.38 \mathrm{eV}$, corresponding to a strong $n$-doping 

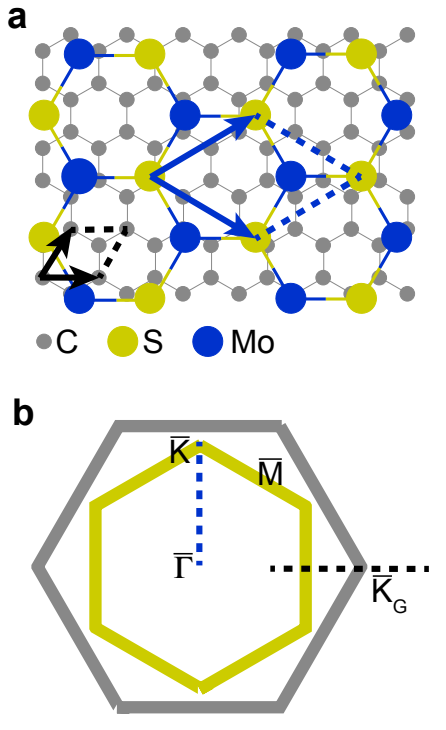

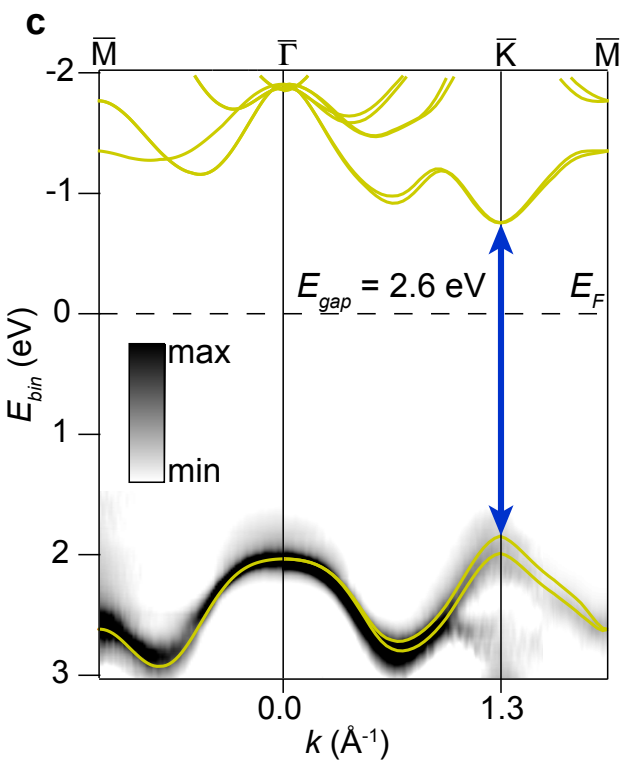

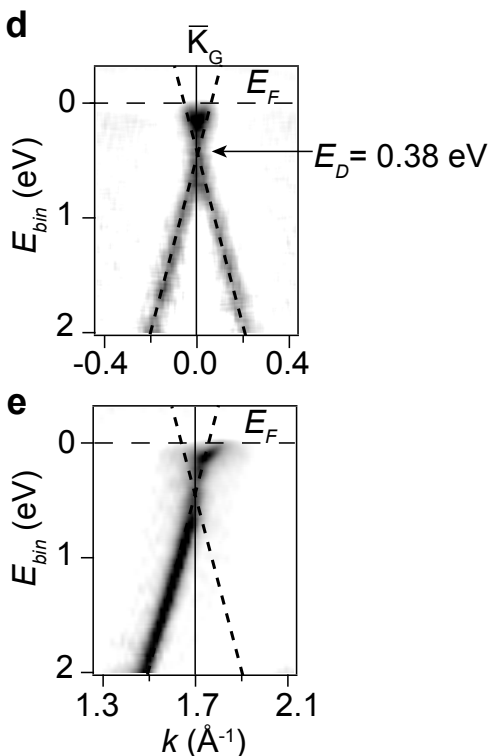

Figure 1: Equilibrium electronic structure of $\mathrm{SL} \mathrm{MoS}_{2}$ on graphene: (a) Alignment of the crystal lattices of epitaxial SL $\mathrm{MoS}_{2}$ on graphene. The unit cells (arrows and dashed lines) are rotated by $30^{\circ}$ with respect to each other. (b) Corresponding BZs for $\mathrm{MoS}_{2}$ (yellow) and graphene (grey). The measurement directions for the TR-ARPES experiments are shown by blue $\left(\mathrm{SL} \mathrm{MoS}_{2}\right)$ and black (graphene) dashed lines. The corner of the graphene BZ is labeled $\bar{K}_{G}$, while the $\mathrm{MoS}_{2}$-related symmetry points do not have an index. (c) Band structure of the upper VB for SL $\mathrm{MoS}_{2}$ measured by ARPES and overlaid with the theoretical VB and $\mathrm{CB}$ (yellow curves) assuming a quasiparticle gap of $2.6 \mathrm{eV}$ for $\mathrm{SL} \mathrm{MoS}_{2} / \mathrm{G}$. (d)-(e) Band structure around the Dirac point $\left(E_{D}\right)$ of graphene with a linear dispersion (black dashed lines) overlaid as a guide to the eye. The cuts in (d) and (e) are perpendicular and parallel to the dashed line in (b), respectively. The cut in (e) corresponds to the measurement direction in TR-ARPES. The second derivative of the photoemission intensity is shown for all the ARPES data.

$\left(n \approx 1.1 \times 10^{13} \mathrm{~cm}^{-2}\right)$ that is similar to previously reported band structure measurements for graphene synthesised by similar methodologies but without the SL MoS $\mathrm{M}_{2}$ film on top. ${ }^{18,20}$

The unoccupied electronic states and band gap for SL MoS 2 are not accessible by ARPES. For the sketches in Figures 1c and 2a, we assume a static band gap of $2.6 \mathrm{eV}$. This value is estimated from the theoretical values of $2.7-2.8 \mathrm{eV}$ for a free-standing layer ${ }^{5,13,21}$ and an environmental screening-induced renormalization of $\approx 0.2 \mathrm{eV}$ that has been observed for the similar system of $\mathrm{MoSe}_{2}$ on bilayer graphene. ${ }^{4}$ We shall later see that a static band gap of this size fits well with our TR-ARPES measurement of the excited state when we extrapolate 

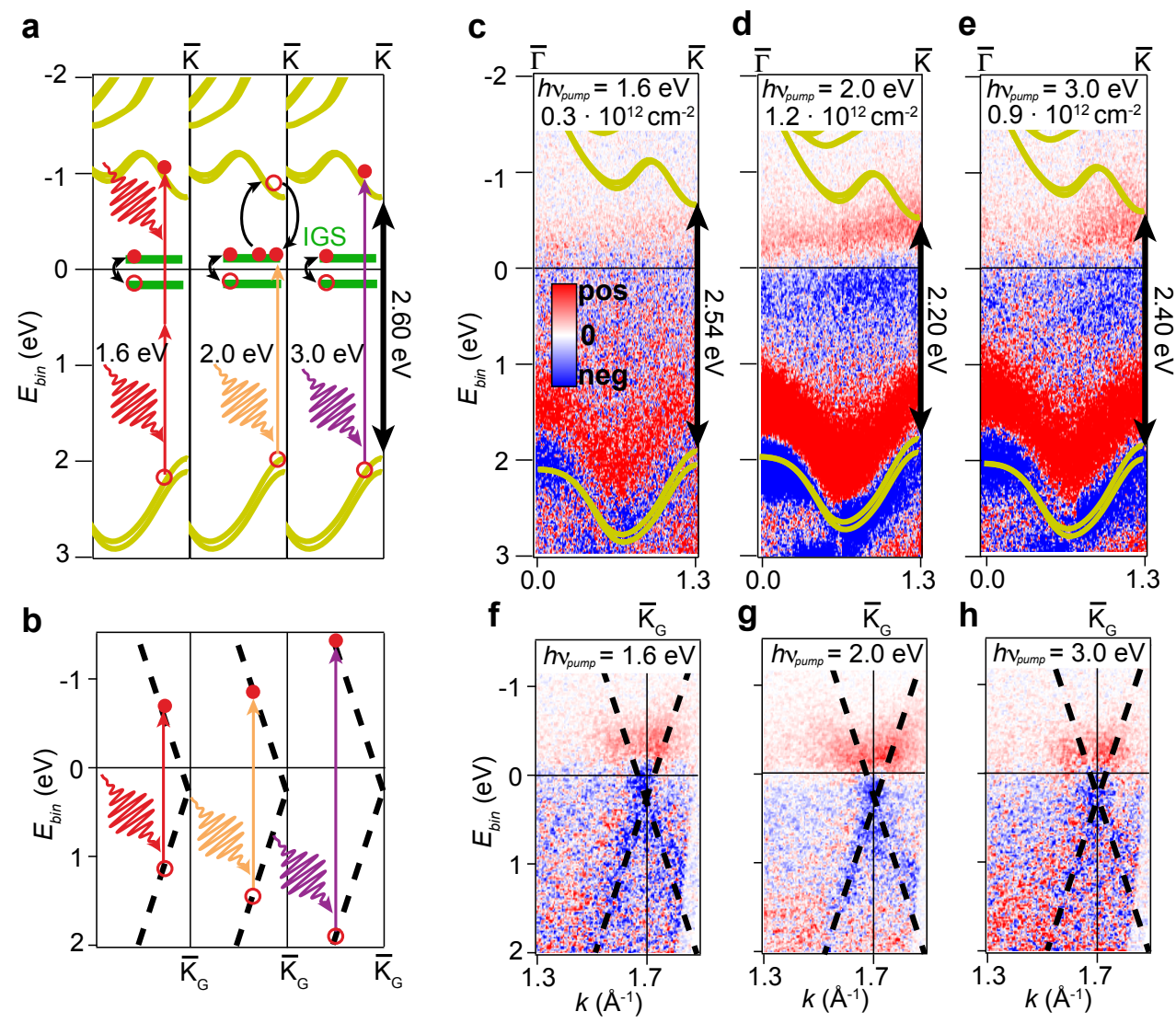

Figure 2: Excitation of a $\mathrm{MoS}_{2} / \mathrm{G}$ heterostructure: (a) Possible electron-hole pair excitation processes in $\mathrm{SL} \mathrm{MoS}_{2}$ for the applied pump energies of $1.6 \mathrm{eV}, 2.0 \mathrm{eV}$ and $3.0 \mathrm{eV}$. When the pump energy is below the band gap, the excitation must happen via higher order multiple photon processes (shown for the $1.6 \mathrm{eV}$ case), the involvement of in-gap states (IGS) (shown for the $2.0 \mathrm{eV}$ case) or exciton dissociation (not shown). The equilibrium quasiparticle gap energy is assumed to be $2.60 \mathrm{eV}$. (b) Corresponding excitations in graphene. Direct transitions are allowed for all photon energies considered. (c)-(e) Intensity difference spectra of the $\mathrm{MoS}_{2}$ bands at peak excitation along the $\bar{\Gamma}-\bar{K}$ direction. Values for the renormalized gap are provided based on the VB shift and assuming a symmetric CB shift. The maximum optically induced hole density is provided in each panel. (f)-(h) Intensity difference in the graphene bands at peak excitation. The fluences are (c), (f) $F=0.7 \mathrm{~mJ} / \mathrm{cm}^{2},(\mathrm{~d}),(\mathrm{g})$ $F=3.0 \mathrm{~mJ} / \mathrm{cm}^{2}$ and (e), (h) $F=1.3 \mathrm{~mJ} / \mathrm{cm}^{2}$.

our measured band gap renormalization to the limit of zero free carrier density. Note that the static band gap renormalization for $\mathrm{SL} \mathrm{MoS}_{2}$ on graphene is substantially smaller than for $\mathrm{MoS}_{2}$ on a truly metallic substrate such as $\mathrm{Au}(111)$ where the band gap is reduced by $\approx 0.9 \mathrm{eV} \cdot 9,10$

Figure 2a shows possible excitation processes in $\mathrm{SL} \mathrm{MoS}_{2}$ during the pump phase of 
the TR-ARPES experiment. For pump pulse photon energies of $h \nu=1.6 \mathrm{eV}$ and $2.0 \mathrm{eV}$ we observe that the sample is excited even though direct transitions from the VB to the $\mathrm{CB}$ are not possible. In these cases, both two-photon absorption or Auger recombination, involving in-gap states, due to defects (illustrated in the left and middle panels of Figure 2a, respectively) may contribute to excitation. For $h \nu=2.0 \mathrm{eV}$ where the energy is resonant with the A exciton in $\mathrm{MoS}_{2}$ we expect that exciton dissociation will also become significant leading to excited free electrons and holes. At $h \nu=3.0 \mathrm{eV}$, direct transitions become possible. For graphene the gapless Dirac cone ensures that direct transitions are possible for all photon energies considered here (Figure 2b). Excitation of electrons from graphene to $\mathrm{MoS}_{2}$ could be possible in all cases, but we do not observe any indication of this in the time dependence of the signals in the two materials as discussed later in connection with Figure 5.

Figures 2c-e and 2f-h show the intensity difference between a spectrum taken before the optical excitation and a spectrum taken at the peak excitation (typically after 40 fs) for the SL $\mathrm{MoS}_{2}$ and graphene bands. The excitation energy and pump fluence for data sets in the same column are identical, so that the resulting effects can be compared directly. For the $\mathrm{MoS}_{2}$ data we provide calculated values of the optically induced hole density. These calculations are discussed in more depth in connection with Figure 4 and in the Supplementary Material. For graphene, the promotion of electrons from the VB to the CB leads to a depletion of photoemission intensity below the Fermi energy $E_{F}$ (blue) and a corresponding increase above $E_{F}$ (red), in the region of the Dirac cone. This behaviour is qualitatively similar to the situation without $\mathrm{MoS}_{2}$ and has been observed for graphene and bilayer graphene for different doping levels. ${ }^{18,22-25}$

For $\mathrm{MoS}_{2}$ the situation is entirely different: While the CB shows an increased population near the conduction band minimum (CBM), at least for Figure 2d-e, the VB not only shows the expected intensity loss (blue) but also a pronounced intensity gain (red), immediately above the blue region. This spectral change in the VB corresponds to an overall intensity loss combined with a shift of the band to lower binding energy. We clearly observe that 


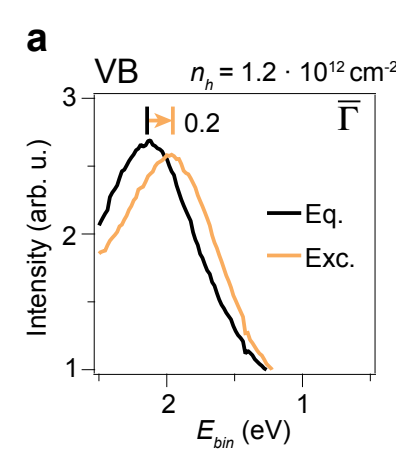

b
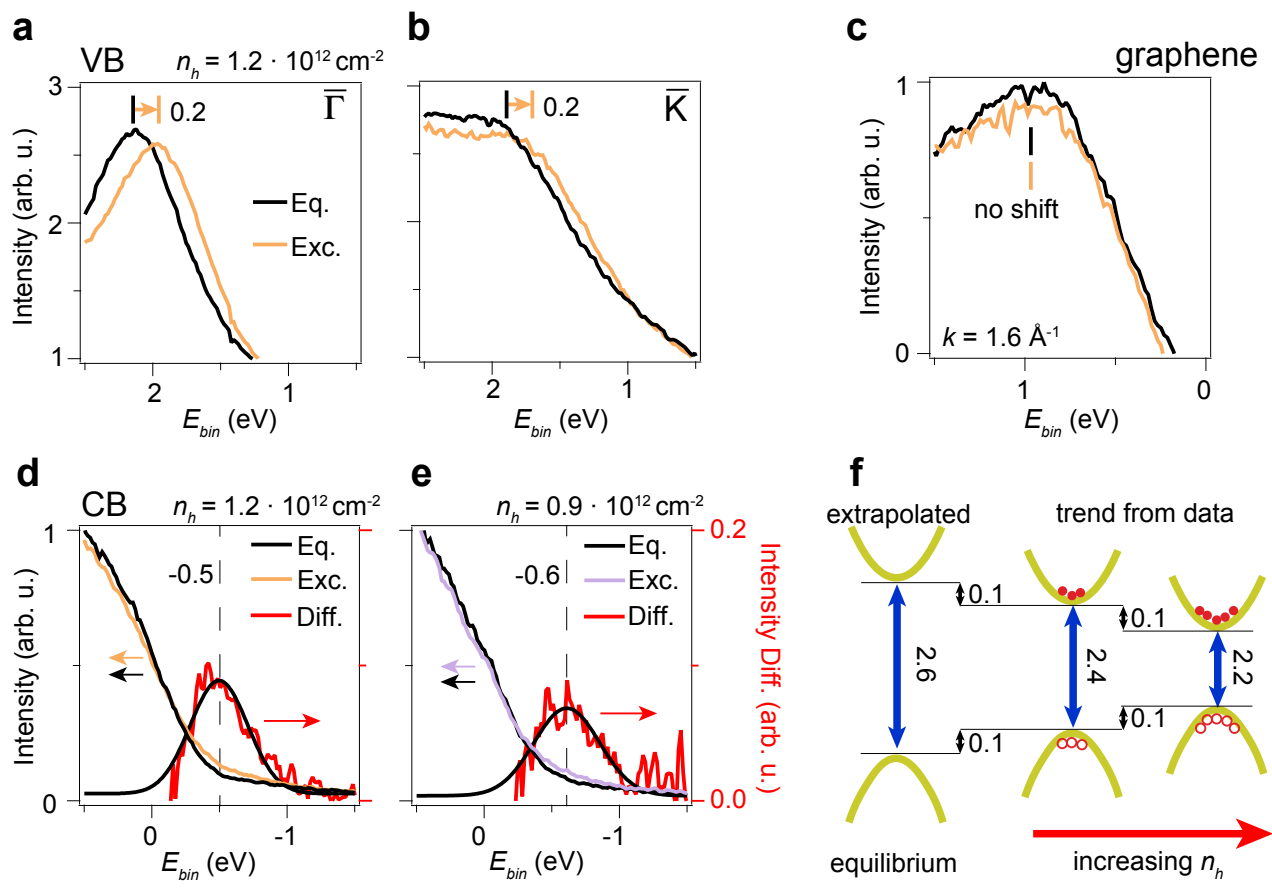

Figure 3: Band shifts and band gap renormalization: (a)-(b) EDC cuts through the data for $\mathrm{MoS}_{2}$ in Figure $2 \mathrm{~d}$ at $\bar{\Gamma}$ and $\bar{K}$, respectively, illustrating a rigid shift and an intensity loss in the VB from the equilibrium (Eq.) to the excited (Exc.) state. (c) Corresponding EDC for graphene, taken from the data in Figure $2 \mathrm{~g}$ showing just an intensity loss but no shift. (d)-(e) EDCs around $\bar{K}$ in the CB region of $\mathrm{MoS}_{2}$ extracted from the data in Figure 2d and 2e, respectively. Curves are displayed in the equilibrium and fully excited cases along with the intensity difference. The peak in the difference is fitted by a Gaussian line shape and the peak value is interpreted as the position of the CBM. (f) Sketch of the observed VB and $\mathrm{CB}$ shifts and corresponding band gap renormalization as a function of free carrier density. The shifts are extrapolated to obtain a band gap renormalization of $2.6 \mathrm{eV}$ in the zero free carrier density limit at equilibrium. All numbers are stated in $\mathrm{eV}$ unless otherwise noted.

these are rigid band shifts that occur exclusively in the $\mathrm{MoS}_{2}$ related states by analyzing energy distribution curve (EDC) cuts of the data at constant $k$. Figure 3a and 3b illustrate the observed rigid shift $(0.2 \mathrm{eV})$ for an optically induced hole density of $1.2 \cdot 10^{12} \mathrm{~cm}^{-2}$ at $\bar{\Gamma}$ and $\bar{K}$, respectively. Similar shifts are observed for all excitations but their size depends on the pump energy and fluence, and Figure 2c-e show data for representative combinations of these parameters. Rigid shifts are shown via EDCs at both $\bar{\Gamma}$ and $\bar{K}$ for additional pump pulse settings in Figure S1g-h.

Such spectral shifts can be induced by space charge or surface photovoltage (SPV) ef- 
fects. ${ }^{26}$ The space charge effect is caused by interactions among the photoelectrons in vacuum, which sets up a charge cloud that propagates away from the sample. The acceleration of photoemitted electrons is consequently changed as they propagate to the detector, leading to shifts in the measured kinetic energy distributions. The SPV effect is caused by excitation of electron-hole pairs in the $\mathrm{SiC}$ substrate, which modifies the band bending and thereby the electrostatic potential at the surface. ${ }^{27}$ This leads to a time-dependent electrostatic force, exerted by the SPV-derived electric field in the SiC substrate, on all photoemitted electrons, which leads to shifts in the measured spectra. In our case these experimental artefacts can be rigorously excluded since they would affect the graphene bands in the same way as the SL $\mathrm{MoS}_{2}$ bands. This is clearly not the case since shifts for graphene are neither observed in the difference plots of Figure 2f-h nor in EDC cuts as in Figure 3c. The timescale of such shifts would resemble the photoelectron propagation time in vacuum, which is several nanoseconds. ${ }^{28}$ While it is not immediately intuitive, both the SPV and space charge effects cause shifts at negative and positive time delays. At negative time delays, where the photoelectron is emitted before the pump pulse arrives, the electric fields due to pump-induced SPV or space charge clouds can affect the propagating electron in vacuum - an effect which can persist in spectra measured hundreds of picoseconds before the optical excitation as shown for graphene on $\mathrm{SiC}$ in Ref. 26. At positive time delays, the SPV decays on a timescale corresponding to the carrier recombination time in SiC. In our case, shifts do not occur for negative time delays and the time dependence of the spectral changes at positive time delays does not resemble the photoelectron propagation time in vacuum or the recombination time of excited carriers in $\mathrm{SiC}$ (the time dependence of the measured band shifts is shown in Figure 5).

While the absolute positions and shifts of the VB states may be determined from the EDCs shown in Figure 3a-b, a corresponding analysis for the CB is limited by the fact that the CB states are not observable in equilibrium, see Figure 3d-e. Hence, we can not directly extract the initial binding energy of the CBM. We do, however, observe indications of excited 
CB states above the Fermi level as an intensity increase around $\bar{K}$ following photoexcitation, at least for a sufficiently strong excitation as is the case in Figures 3d-e. For these datasets we can fit a Gaussian line shape to an EDC at $\bar{K}$, and use the fitted peak position as a CBM estimate. In Figure 2d-e a slight intensity loss is visible immediately below the Fermi level. The spectral weight in this region comes from in-gap defect states. The optical excitation produces a distribution of hot electrons (and holes) in these states, as illustrated in all panels of Figure 2a. The broadening of the Fermi edge that accompanies this hot carrier population is directly visible in Figure 3d-e. The surplus of intensity above the Fermi level due to these defect states contributes as a background to the main signal coming from the excited population in the CBM. From comparing the CBM position as a function of photon energy and fluence in Figures 3d-e, we find that the CBM shift is the same size but opposite direction as the observed shifts of the VBM in Figures 3a-b and S1g-j. Even though the uncertainties are large in this analysis, extrapolating such symmetric VBM and CBM shifts to zero excited carrier density leads to an equilibrium quasiparticle gap consistent with a value of $2.6 \mathrm{eV}$ as illustrated in Figure 3f. Note that the overlaid calculated $\mathrm{CB}$ and VB in Figure 2c-e have been rigidly shifted in accordance with this analysis.

In order to determine the optically induced number of holes that accompanies the band shifts, we apply an EDC-based analysis of the intensity loss in the VB introduced in Figure 4(a)-(b). As shown for EDCs around the $\bar{\Gamma}$ (Figure 4a) and $\bar{K}$ (Figure $4 \mathrm{~b}$ ) points for the data in Figure 2d, we perform a fit with a Lorentzian on a linear background. The background is allowed to vary for each momentum point due to tails from nearby graphene bands, however, the background is held fixed as a function of time. The data taken before the optical excitation are normalized such that the Lorentzian integrates up to the number of electrons (2), which occupy a single momentum state in equilibrium. The integrated Lorentzian after the optical excitation can then be used to determine the number of holes in the $k$-range of the EDC. Applying this procedure for the data along $\bar{\Gamma}-\bar{K}$ results in the number of holes $N_{h}$ at peak excitation as a function of $k$, as shown in Figure 4c-h for the 

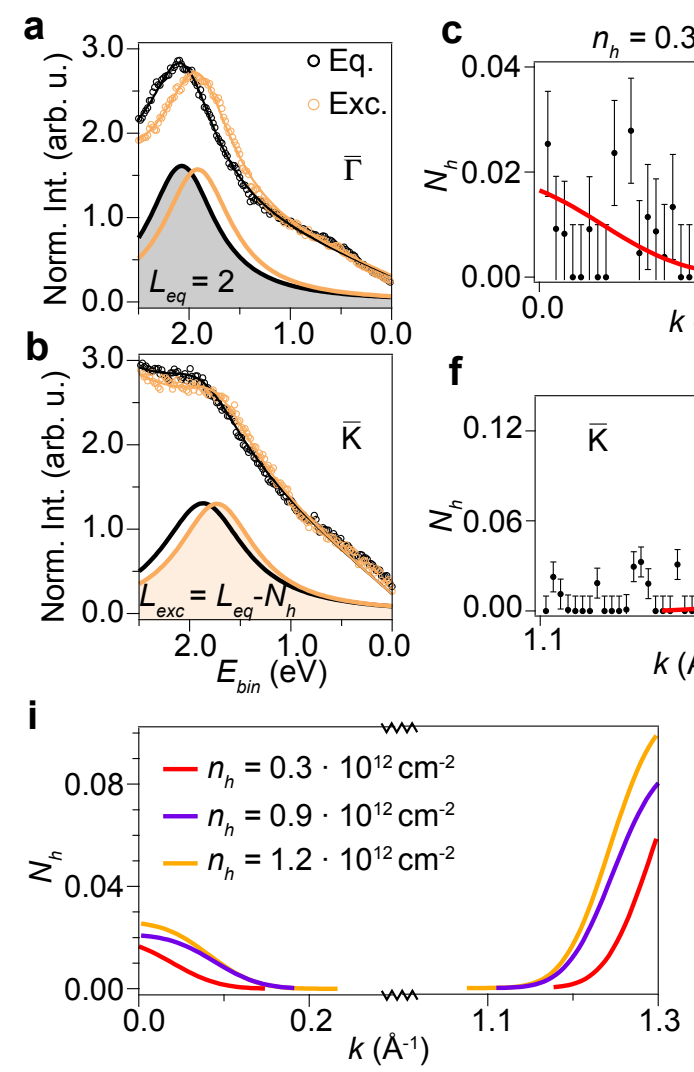
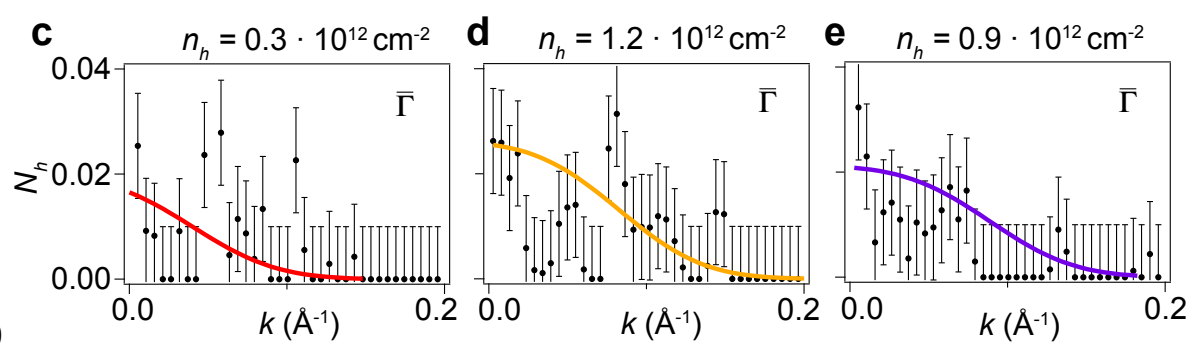

g

h

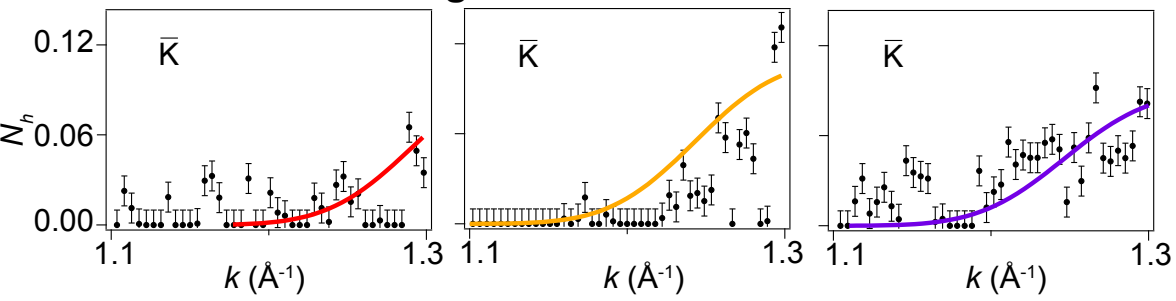

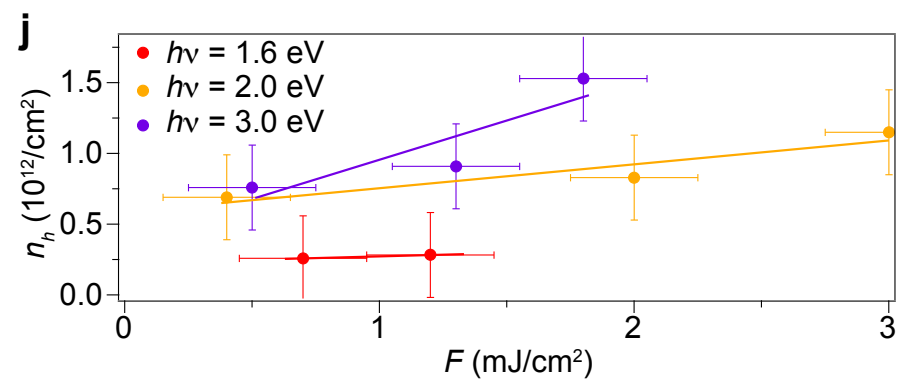

Figure 4: Analysis of optically induced hole density in the SL $\mathrm{MoS}_{2}$ layer: (a)-(b) EDCs (markers) at $\bar{\Gamma}$ and $\bar{K}$, plotted for a time delay before the optical excitation (black) and at peak excitation (orange) for the data in Figure 2d. Fits to a linear background and a single Lorentzian line shape are shown by lines and the Lorentzian parts are plotted individually. The data are normalized such that the Lorentzian area $L_{e q}$ before excitation integrates up to 2 electrons $\left(L_{e q}=2\right.$, shown for $\bar{\Gamma}$ in (a)). The number of generated holes in the state $N_{h}$ can then be determined from the Lorentzian area in the excited case $L_{\text {exc }}$ by $N_{h}=L_{e q}-L_{\text {exc }}$ (shown for $\bar{K}$ in (b)). (c)-(e) Hole profiles $N_{h}(k)$ around $\bar{\Gamma}$ and (f)-(h) around $\bar{K}$. The markers are the experimental data obtained from the analysis in (a)-(b). Smooth curves are fits to a distribution function. (i) Fitted distribution functions from (c)-(h) shown for clarity. (j) Calculated hole density for $\mathrm{SL} \mathrm{MoS}_{2}$ at peak excitation for different combinations of pump energy and fluence. Lines through the data points have been added as a guide to the eye.

same pump pulse settings as the corresponding Figure 2c-e. The overall intensity loss in the EDCs following excitation is comparable to the level of the noise, however, in the data $N_{h}$ is strongly peaked around the VB maxima at $\bar{\Gamma}$ and $\bar{K}$ at any given time and clearly approaches zero away from the band maxima at $\bar{\Gamma}$ and $\bar{K}$. This behavior agrees with the expectation, that the holes reach a quasi-equilibrium on a much faster time scale than our 
time resolution of $40 \mathrm{fs}$, leading to transient Fermi-Dirac like distributions near the band extrema. Fits of such functions (see Figure S3c for an example of a raw fitting function) to all data are shown in each panel of Figure 4c-h and combined in Figure 4i for ease of comparison. The number of holes never exceeds $10 \%$ of the available states.

Because of the hole distribution's strong energy dependence and the tendency to even an anisotropic distribution out on a short time scale, ${ }^{29}$ it is reasonable to assume an isotropic distribution around the local band maxima. The total hole density $n_{h}$ in the VB can then be calculated and the result is shown at peak excitation in Figure $4 \mathrm{j}$. As expected, $n_{h}$ depends on both the pump photon energy and fluence. The lines added to each set of points in Figure $4 \mathrm{j}$ have been included as a guide to the eye to compare the overall magnitude and trend of hole density with fluence for a given pump pulse energy. The excited hole density is expected to extrapolate to zero and thus be a highly nonlinear function with fluence. A complete understanding of this dependence is beyond the scope of this work. Furthermore, a simple estimate using the pump pulse fluences and photon energies applied here would lead to excited hole densities on the order of $10^{14}-10^{15} \mathrm{~cm}^{-2}$ using the absorbance of 5$10 \%$ for SL $\mathrm{MoS}_{2} \cdot{ }^{30}$ The much smaller hole densities we obtain are consistent with previous TR-ARPES studies on graphene, ${ }^{18,22}$ and can be attributed to a combination of effects such as Pauli blocking, recombination of carriers on faster timescales than we probe, diffusion of excited carriers out of the probed area of the sample, as well as imperfect overlap of pump and probe beams. Note that Figures 2 and 4 only provide values at peak excitation but the energy shifts and hole densities are analyzed for entire sets of data with different delay times between pump and probe pulse as shown in Figure S1i-j. This allows us to correlate hole densities with the corresponding band gap reduction for a wide range of parameters and conditions.

In Figure 5a we analyze the dynamical band gap reduction as a function of delay time between the pump and probe pulse for the three data sets from Figure 2c-e. The largest effect of a nearly $0.4 \mathrm{eV}$ shrinkage is seen for the data taken with a pump pulse energy 

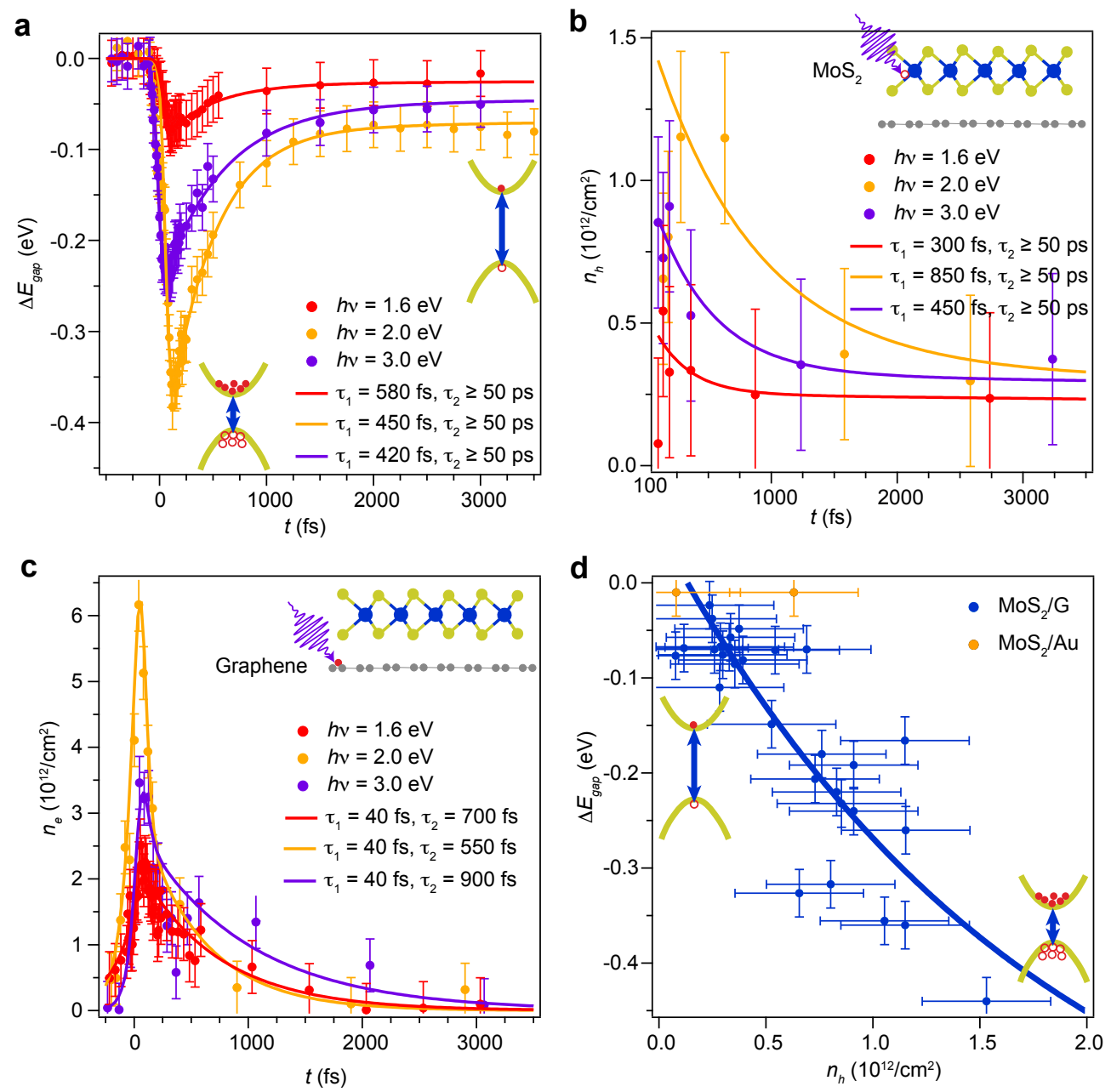

Figure 5: Tuning the band gap via optically induced carrier densities and substrate screening: (a) Time-dependent band gap renormalization for the data sets in Figure 2c-e. The lines correspond to an exponential rising edge followed by a double exponential decay with the decay time constants given in the figure. (b) Hole density as a function of delay time for the same data sets. Again, the two time constants for a double exponential decay are given. The time scale here begins at $100 \mathrm{fs}$ because $n_{h}$ is undefined before the peak optical excitation is reached. For the data after the peak excitation the time-delays have been binned over several time delay points. (c) Time-dependent hole density for the underlying graphene layer, extracted from the data sets in Figure 2f-h. The time constants for a double exponential decay are given. (d) Plot of the total band gap renormalization versus the experimentally extracted hole densities. A comparison with similar data obtained using a $\mathrm{Au}(111)$ substrate is included (Data from Ref. 9). The blue curve is merely a guide to the eye.

of $2 \mathrm{eV}$, which also has the highest fluence and results in the highest hole density at peak excitation (see Figure 2d). The decay of the band gap shrinkage is fitted with a double exponential function and the characteristic decay times are similar for the three data sets 
$(\approx 500 \mathrm{fs}$ and $\approx 50 \mathrm{ps})$. Note that the time constant of $50 \mathrm{ps}$ for the slow component is a lower estimate. Figure 5b shows the corresponding time dependence of the hole density. A double exponential fit to these results in similar time constants but the uncertainties on the individual data points are very large, even when wider ranges of time delays are binned into a single data point. Nevertheless, the similar time constants strongly support the interpretation that the observed band narrowing is induced by the excited carriers. The presence of two characteristic decay times is also consistent with all-optical experiments. ${ }^{31}$

Figure 5c shows the time-dependent electron density in the graphene layer for the three data sets shown in Figure $2 \mathrm{f}-\mathrm{h}$, illustrating that the carrier dynamics is entirely different from the dynamics of the band gap reduction and hole density in the SL MoS 2 . Here a double exponential fit results in a fast decay time that is more than an order of magnitude shorter than that observed for SL $\mathrm{MoS}_{2}$. Since the optically induced electrons in graphene thermalize within our time resolution, it is possible to estimate the transient electron density from the electronic temperature. The extracted time-dependence of the electron density corresponds well to that observed in previous experiments without the SL $\mathrm{MoS}_{2}$ on top. ${ }^{18,25}$ Even though we have regions of bare graphene within the probed area of the sample due to our $\mathrm{MoS}_{2}$ coverage of approximately $0.55 \mathrm{ML}^{19}$ we also expect some signal from the graphene under the $\mathrm{MoS}_{2} \cdot{ }^{32}$ The observation of a single time constant suggests that the graphene dynamics are at most weakly affected by the overlaid $\mathrm{MoS}_{2}$.

Finally, Figure 5d summarizes the key result of this paper, the band gap shrinkage as a function of excited hole density, combining data taken with different excitation energies and fluences. These results can be compared to a recent prediction by Steinhoff et al. which gives a somewhat larger shrinkage but with the same order of magnitude as observed here $\left(\approx 0.5 \mathrm{eV}\right.$ for a carrier density of $\left.\approx 10^{12} \mathrm{~cm}^{-2}\right) .{ }^{5}$ The discrepancy between experiment and theory can easily be accounted for by the uncertainties in the determination of the carrier concentration and the role of the underlying graphene, which is not present in the calculation.

Similar carrier-induced renormalization effects have been observed for quasi-free-standing 
$\mathrm{MoS}_{2}$ on $\mathrm{SiO}_{2}$, either by optical or electrical doping ${ }^{6,7}$ and for surface doping of bulk $\mathrm{WSe}_{2} \cdot{ }^{33}$ However, the effect is nearly completely suppressed when $\mathrm{MoS}_{2}$ is placed in a strongly screening environment such as on a metallic $\mathrm{Au}(111)$ substrate. ${ }^{9,10}$ To illustrate this, we have added the result of a corresponding analysis, using data for $\mathrm{MoS}_{2} / \mathrm{Au}(111)$ from Ref. 9. In this case (orange data points in Figure 5d) the band shift remains below $10 \mathrm{meV}$, even for high induced carrier densities. Also, the typical decay times for excited carriers ( $\approx 50 \mathrm{fs}$ ) are much faster than observed here or for $\mathrm{MoS}_{2}$ on $\mathrm{SiO}_{2} \cdot{ }^{6,31}$ Our results are reminiscent of TR-ARPES studies on the strongly correlated metallic TMDC $1 \mathrm{~T}-\mathrm{TiSe}_{2} \cdot{ }^{34,35}$ This system exhibits a charge ordered state, which is completely removed due to additional screening from optically excited free carriers. ${ }^{34}$ This scenario is consistent with our interpretation of a band gap reduction in SL $\mathrm{MoS}_{2}$ due to the build-up of screening via laser-induced free carriers.

\section{Conclusion}

As our results show, the static screening of charge carriers in $\mathrm{SL} \mathrm{MoS}_{2}$ in a heterostructure with graphene is sufficiently weak that a significant band gap renormalization can be induced by optically excited carriers. On a fundamental level, this suggests that the degree of control of the electronic structure of such heterostructures extends beyond the substrate-induced renormalization of the static band gap of the $2 \mathrm{D}$ semiconductor to the dynamic tuneability of the band gap and the electron dynamics. This behavior is essential to take into account in optical applications where the change of the resonance condition on ultrafast time scales may pose a serious challenge, e.g. in the design of an actual optical cavity. On the other hand, one could imagine optoelectronic devices based on a heterostructure with different band alignments in the constituent materials where ultrafast light-induced switching of the band offsets could be used as a means to control the separation of excitons and free charge carriers. 


\section{Materials and Methods}

\section{Sample preparation}

The $\mathrm{MoS}_{2} / \mathrm{G}$ heterostructure was synthesized on a $6 \mathrm{H}-\mathrm{SiC} 2$ in. wafer (TanKeBlue Semiconductor Co. Ltd., $n$-type doping, 0.02-0.10 $\Omega \cdot \mathrm{cm})$. The epitaxial graphene layer was grown first on the Si terminated (0001) face by direct current annealing of the $6 \mathrm{H}-\mathrm{SiC}$ substrate in a dedicated ultra-high vacuum (UHV) graphene growth chamber with a base pressure of $5 \times 10^{-10}$ mbar. The dangling Si bonds of the substrate were saturated by a carbon buffer

layer with a $(6 \sqrt{3} \times 6 \sqrt{3}) R 30^{\circ}$ periodicity under the graphene layer. $\mathrm{MoS}_{2}$ was then grown on top of the graphene by van der Waals epitaxy in a separate UHV chamber. In this procedure the Mo was initially deposited from an electron beam evaporator (Oxford Instruments) in a $\mathrm{H}_{2} \mathrm{~S}$ atmosphere of $\approx 1 \times 10^{-5}$ mbar. After deposition the sample was annealed for $\approx 1$ hour at $1050 \mathrm{~K}$ in the $\mathrm{H}_{2} \mathrm{~S}$ environment, which resulted in nanosized islands of $\mathrm{MoS}_{2}$ on the graphene. Repeated cycles of this process lead to the formation of a SL $\mathrm{MoS}_{2}$ film with an optimum coverage around 0.55 ML before bilayer $\mathrm{MoS}_{2}$ islands nucleated, as determined by scanning tunneling microscopy. Further details on the growth are provided in Ref. 19.

\section{ARPES}

The equilibrium electronic structure of the $\mathrm{MoS}_{2} / \mathrm{G}$ sample was determined by ARPES at the SGM-3 UHV end-station of the synchrotron radiation source ASTRID2 in Aarhus, Denmark. ${ }^{36}$ The sample was initially annealed to $500 \mathrm{~K}$ to remove adsorbed species. The ARPES data was collected at a photon energy of $70 \mathrm{eV}$ over a significant portion of the $\mathrm{MoS}_{2}$ and graphene Brillouin zones $(\mathrm{BZs})$ in order to determine the location of the band extrema of the two materials and their relative orientation with respect to each other. This information was used to align the sample in the TR-ARPES experiments. The sample temperature was kept at $70 \mathrm{~K}$ and the total energy- and angular-resolution were set to $20 \mathrm{meV}$ and $0.2^{\circ}$, 
respectively.

\section{TR-ARPES}

The same sample that was measured by ARPES was then transported in an evacuated tube pumped down below $1 \times 10^{-9}$ mbar to the Artemis facility, Rutherford Appleton Laboratory in Harwell, UK for TR-ARPES measurements. ${ }^{37}$ Here it was placed in the TRARPES UHV end-station and annealed to $500 \mathrm{~K}$ in order to remove any adsorbed surface contaminants. The sample temperature was kept at $50 \mathrm{~K}$ using a liquid helium cryostat during measurements.

The pump and probe pulses used for acquiring TR-ARPES spectra were generated using a $1 \mathrm{kHz}$ Ti:sapphire amplified laser system with a wavelength of $785 \mathrm{~nm}$, a pulse duration of $30 \mathrm{fs}$ and an energy per pulse of $12 \mathrm{~mJ}$. The band structures of $\mathrm{MoS}_{2}$ and graphene were measured along their $\bar{\Gamma}-\bar{K}$ directions using high harmonic probe pulses of $h \nu=25 \mathrm{eV}$, which were generated by focusing a part of the laser energy on a pulsed jet of argon gas. The remaining laser energy was applied to drive an optical parametric amplifier (HE-Topas) followed by a frequency mixing stage. This provided tunable pump pulses with wavelengths centered at $408 \mathrm{~nm}(3.0 \mathrm{eV}), 615 \mathrm{~nm}(2.0 \mathrm{eV})$ and $784 \mathrm{~nm}(1.6 \mathrm{eV})$, which were used to optically excite the sample. The fluence of the pump pulse was kept in the range 0.4$3.0 \mathrm{~mJ} / \mathrm{cm}^{2}$, and the maximum used for any of the three applied wavelengths was kept below the onset where significant energy broadening and shifts of the measured spectra started to

occur due to pump-induced space-charge effects. ${ }^{26}$ The beams were polarized such that the pump pulse was $s$-polarized and the probe pulse was $p$-polarized.

The time delays of pump and probe pulses were varied using a mechanical delay line. We applied two modes of acquisition: (1) A few-delay-point mode where we measured the spectra over 3-6 time delay points around peak optical excitation but acquired these cumulatively up to 2500 times in order to optimize signal-to-noise ratio. (2) A many-delay-point mode, 
where we measured the spectra over 50-60 time delay points cumulatively up to 1000 times. Acquisition times of the few-delay-point mode were up to $3 \mathrm{~h}$, which enabled us to acquire data at 3-4 different pump fluence settings per pump wavelength. In the many-delay-point mode acquisition times were up to $20 \mathrm{~h}$ per dataset, which limited the number of experimental parameters that we could vary in such experiments, but allowed us to capture the detailed time dependence. Throughout this work we have defined $t=0$ to coincide with the middle

of the rising edge in the measured spectra following optical excitation of the sample. The energy-, angular- and time-resolution were $400 \mathrm{meV}, 0.3^{\circ}$ and $40 \mathrm{fs}$, respectively.

\section{Acknowledgement}

We thank Phil Rice for technical support during the Artemis beamtime. We gratefully acknowledge funding from the VILLUM foundation, the Lundbeck foundation, EPSRC (Grant Nos. EP/I031014/1 and EP/L505079/1), The Royal Society and the Swiss National Science Foundation (NSF). Ph. H. and S. U. acknowledge financial support from the Danish Council for Independent Research, Natural Sciences under the Sapere Aude program (Grant Nos. DFF-4002-00029 and DFF-4090-00125). Access to the Artemis Facility was funded by STFC.

\section{Supporting Information Available}

Supporting online information is available with details on the analysis of band shifts, conduction band position, $\mathrm{MoS}_{2}$ hole density calculation and graphene electron density calculation. This material is available free of charge via the Internet at http://pubs.acs.org/.

\section{References}

1. Geim, A. K.; Grigorieva, I. V. Van der Waals Heterostructures. Nature 2013, 499, 419. 
2. Georgiou, T.; Jalil, R.; Belle, B. D.; Britnell, L.; Gorbachev, R. V.; Morozov, S. V.; Kim, Y.-J.; Gholinia, A.; Haigh, S. J.; Makarovsky, O.; Eaves, L.; Ponomarenko, L. A.; Geim, A. K.; Novoselov, K. S.; Mishchenko, A. Vertical Field-Effect Transistor Based on Graphene- $\mathrm{WS}_{2}$ Heterostructures for Flexible and Transparent Electronics. Nat. Nanotechnol. 2013, 8, 100 .

3. Roy, K.; Padmanabhan, M.; Goswami, S.; Sai, T. P.; Ramalingam, G.; Raghavan, S.; Ghosh, A. Graphene-MoS 2 Hybrid Structures for Multifunctional Photoresponsive Memory Devices. Nat. Nanotechnol. 2013, 8, 826.

4. Ugeda, M. M.; Bradley, A. J.; Shi, S.-F.; da Jornade, F. H.; Zhang, Y.; Qiu, D. Y.; Ruan, W.; Mo, S.-K.; Hussain, Z.; Shen, Z.-X.; Wang, F.; Louie, S. G.; Crommie, M. F. Giant Bandgap Renormalization and Excitonic Effects in a Monolayer Transition Metal Dichalcogenide Semiconductor. Nat. Mater. 2014, 13, 1091.

5. Steinhoff, A.; Rösner, M.; Jahnke, F.; Wehling, T. O.; Gies, C. Influence of Excited Carriers on the Optical and Electronic Properties of $\mathrm{MoS}_{2}$. Nano Lett. 2014, 14, 3743.

6. Chernikov, A.; Ruppert, C.; Hill, H. M.; Rigosi, A. F.; Heinz, T. F. Population Inversion and Giant Bandgap Renormalization in Atomically Thin $\mathrm{WS}_{2}$ Layers. Nat. Photonics 2015, 9, 466 .

7. Chernikov, A.; van der Zande, A. M.; Hill, H. M.; Rigosi, A. F.; Velauthapillai, A.; Hone, J.; Heinz, T. F. Electrical Tuning of Exciton Binding Energies in Monolayer $\mathrm{WS}_{2}$. Phys. Rev. Lett. 2015, 115, 126802.

8. Pogna, E. A. A.; Marsili, M.; Fazio, D. D.; Conte, S. D.; Manzoni, C.; Sangalli, D.; Yoon, D.; Lombardo, A.; Ferrari, A. C.; Marini, A.; Cerullo, G.; Prezzi, D. Photo-Induced Bandgap Renormalization Governs the Ultrafast Response of Single-Layer $\mathrm{MoS}_{2}$. ACS Nano 2016, 10, 1182. 
9. Grubišić Čabo, A.; Miwa, J. A.; Grønborg, S. S.; Riley, J. M.; Johannsen, J. C.; Cacho, C.; Alexander, O.; Chapman, R. T.; Springate, E.; Grioni, M.; Lauritsen, J. V.; King, P. D. C.; Hofmann, P.; Ulstrup, S. Observation of Ultrafast Free Carrier Dynamics in Single Layer $\mathrm{MoS}_{2}$. Nano Lett. 2015, 15, 5883.

10. Bruix, A.; Miwa, J. A.; Hauptmann, N.; Wegner, D.; Ulstrup, S.; Grønborg, S. S.; Sanders, C. E.; Dendzik, M.; Grubišić Čabo, A.; Bianchi, M.; Lauritsen, J. V.; Khajetoorians, A. A.; Hammer, B.; Hofmann, P. Single-Layer $\mathrm{MoS}_{2}$ on $\mathrm{Au}(111)$ : Band Gap Renormalization and Substrate Interaction. Phys. Rev. B 2016, 93, 165422.

11. Mak, K.; Lee, C.; Hone, J.; Shan, J.; Heinz, T. Atomically Thin $\mathrm{MoS}_{2}$ : A New DirectGap Semiconductor. Phys. Rev. Lett. 2010, 105, 136805.

12. Splendiani, A.; Sun, L.; Zhang, Y.; Li, T.; Kim, J.; Chim, C.-Y.; Galli, G.; Wang, F. Emerging Photoluminescence in Monolayer $\mathrm{MoS}_{2}$. Nano Lett. 2010, 10, 1271.

13. Qiu, D. Y.; da Jornada, F. H.; Louie, S. G. Optical Spectrum of $\mathrm{MoS}_{2}$ : Many-Body Effects and Diversity of Exciton States. Phys. Rev. Lett. 2013, 111, 216805.

14. Kalt, H.; Rinker, M. Band-Gap Renormalization in Semiconductors with Multiple Inequivalent Valleys. Phys. Rev. B 1992, 45, 1139.

15. Tränkle, G.; Leier, H.; Forchel, A.; Haug, H.; Ell, C.; Weimann, G. Dimensionality Dependence of the Band-Gap Renormalization in Two- and Three-Dimensional ElectronHole Plasmas in GaAs. Phys. Rev. Lett. 1987, 58, 419.

16. Das Sarma, S.; Jalabert, R.; Yang, S.-R. E. Band-Gap Renormalization in Semiconductor Quantum Wells. Phys. Rev. B 1990, 41, 8288.

17. Jin, C.; Rasmussen, F. A.; Thygesen, K. S. Tuning the Schottky Barrier at the Graphene/MoS 2 Interface by Electron Doping: Density Functional Theory and ManyBody Calculations. J. Phys. Chem. C 2015, 119, 19928. 
18. Johannsen, J. C.; Ulstrup, S.; Crepaldi, A.; Cilento, F.; Zacchigna, M.; Miwa, J. A.; Cacho, C.; Chapman, R. T.; Springate, E.; Fromm, F; Raidel, C.; Seyller, T.; King, P. D. C.; Parmigiani, F.; Grioni, M.; Hofmann, P. Tunable Carrier Multiplication and Cooling in Graphene. Nano Lett. 2015, 15, 326.

19. Miwa, J. A.; Dendzik, M.; Grønborg, S. S.; Bianchi, M.; Lauritsen, J. V.; Hofmann, P.; Ulstrup, S. Van der Waals Epitaxy of Two-Dimensional $\mathrm{MoS}_{2}$-Graphene Heterostructures in Ultrahigh Vacuum. ACS Nano 2015, 9, 6502.

20. Bostwick, A.; Ohta, T.; Seyller, T.; Horn, K.; Rotenberg, E. Quasiparticle Dynamics in Graphene. Nat. Phys. 2007, 3, 36.

21. Hüser, F.; Olsen, T.; Thygesen, K. S. How Dielectric Screening in Two-Dimensional Crystals Affects the Convergence of Excited-State Calculations: Monolayer $\mathrm{MoS}_{2}$. Phys. Rev. B 2013, 88, 245309.

22. Johannsen, J. C.; Ulstrup, S.; Cilento, F.; Crepaldi, A.; Zacchigna, M.; Cacho, C.; Turcu, I. C. E.; Springate, E.; Fromm, F.; Raidel, C.; Seyller, T.; Parmigiani, F.; Grioni, M.; Hofmann, P. Direct View of Hot Carrier Dynamics in Graphene. Phys. Rev. Lett. 2013, 111, 027403.

23. Gierz, I.; Petersen, J. C.; Mitrano, M.; Cacho, C.; Turcu, I. C. E.; Springate, E.; Stöhr, A.; Köhler, A.; Starke, U.; Cavalleri, A. Snapshots of Non-Equilibrium Dirac Carrier Distributions in Graphene. Nat. Mater. 2013, 12, 1119.

24. Ulstrup, S.; Johannsen, J. C.; Cilento, F.; Miwa, J. A.; Crepaldi, A.; Zacchigna, M.; Cacho, C.; Chapman, R. T.; Springate, E.; Mammadov, S.; Fromm, F.; Raidel, C.; Seyller, T.; Parmigiani, F.; Grioni, M.; King, P. D. C.; Hofmann, P. Ultrafast Dynamics of Massive Dirac Fermions in Bilayer Graphene. Phys. Rev. Lett. 2014, 112, 257401.

25. Someya, T.; Fukidome, H.; Ishida, Y.; Yoshida, R.; Iimori, T.; Yukawa, R.; Akikubo, K.; Yamamoto, Sh.; Yamamoto, S.; Yamamoto, T.; Kanai, T.; Funakubo, K.; Suemitsu, M.; 
Itatani, J.; Komori, F.; Shin, S.; Matsuda, I. Observing Hot Carrier Distribution in an n-Type Epitaxial Graphene on a SiC Substrate. Appl. Phys. Lett. 2014, 104, 161103.

26. Ulstrup, S.; Johannsen, J. C.; Cilento, F.; Crepaldi, A.; Miwa, J. A.; Zacchigna, M.; Cacho, C.; Chapman, R. T.; Springate, E.; Fromm, F.; Raidel, C.; Seyller, T.; King, P. D. C.; Parmigiani, F.; Grioni, M.; Hofmann, P. Ramifications of Optical Pumping on the Interpretation of Time-Resolved Photoemission Experiments on Graphene. J. Electron Spectrosc. Relat. Phenom. 2015, 200, 340.

27. Yang, S.-L.; Sobota, J.; Kirchmann, P.; Shen, Z.-X. Electron Propagation from a PhotoExcited Surface: Implications for Time-Resolved Photoemission. Appl. Phys. A: Mater. Sci. Process. 2014, 116, 85-90.

28. Tanaka, S. Utility and Constraint on the use of Pump-Probe Photoelectron Spectroscopy for Detecting Time-Resolved Surface Photovoltage. J. Electron Spectrosc. Relat. Phenom. 2012, 185, $152-158$.

29. Mittendorff, M.; Winzer, T.; Malic, E.; Knorr, A.; Berger, C.; de Heer, W. A.; Schneider, H.; Helm, M.; Winnerl, S. Anisotropy of Excitation and Relaxation of Photogenerated Charge Carriers in Graphene. Nano Lett. 2014, 14, 1504.

30. Bernardi, M.; Palummo, M.; Grossman, J. C. Extraordinary Sunlight Absorption and One Nanometer Thick Photovoltaics using Two-Dimensional Monolayer Materials. Nano Lett. 2013, 13, 3664-3670.

31. Wang, H.; Zhang, C.; Rana, F. Ultrafast Dynamics of Defect-Assisted Electron-Hole Recombination in Monolayer $\mathrm{MoS}_{2}$. Nano Lett. 2015, 15, 339.

32. Zhang, Y.; Chang, T.; Zhou, B.; Cui, Y.; Yan, H.; Liu, Z.; Schmitt, F.; Lee, J.; Moore, R.; Chen, Y.; Lin, H.; Jeng, H.-T.; Mo, S.-K.; Hussain, Z.; Bansil, A.; Shen, Z. X. Direct Observation of the Transition from Indirect to Direct Bandgap in Atomically Thin Epitaxial $\mathrm{MoSe}_{2}$. Nat. Nanotechnol. 2014, 9, 111-115. 
33. Riley, J. M.; Meevasana, W.; Bawden, L.; Asakawa, M.; Takayama, T.; Eknapakul, T.; Kim, T. K.; Hoesch, M.; Mo, S. K.; Takagi, H.; Sasagawa, T.; Bahramy, M. S.; King, P. D. C. Negative Electronic Compressibility and Tunable Spin Splitting in $\mathrm{WSe}_{2}$. Nat. Nanotechnol. 2015, 10, 1043.

34. Rohwer, T.; Hellmann, S.; Wiesenmayer, M.; Sohrt, C.; Stange, A.; Slomski, B.; Carr, A.; Liu, Y.; Avila, L. M.; Kallane, M.; Mathias, S.; Kipp, L.; Rossnagel, K.; Bauer, M. Collapse of Long-Range Charge Order Tracked by Time-Resolved Photoemission at High Momenta. Nature 2011, 471, 490-493.

35. Hellmann, S.; Rohwer, M.; Kalläne, M.; Hanff, K.; Sohrt, C.; Stange, A.; Carr, A.; Murnane, M. M.; Kapteyn, H. C.; Kipp, L.; Bauer, M.; Rossnagel, K. Time-Domain Classification of Charge-Density-Wave Insulators. Nat. Commun. 2012, 3, 1069.

36. Hoffmann, S. V.; Søndergaard, C.; Schultz, C.; Li, Z.; Hofmann, P. An UndulatorBased Spherical Grating Monochromator Beamline for Angle-Resolved Photoemission Spectroscopy. Nucl. Instrum. Methods Phys. Res., Sect. A 2004, 523, 441.

37. Frassetto, F.; Cacho, C.; Froud, C. A.; Turcu, I. C. E.; Villoresi, P.; Bryan, W. A.; Springate, E.; Poletto, L. Single-Grating Monochromator for Extreme-Ultraviolet Ultrashort Pulses. Opt. Express 2011, 19, 19169. 